\title{
Wachstumsmarkt Pflege
}

Dörte Heger

Inhaltsverzeichnis

10.1 Einleitung -146

10.2 Langzeitpflege in Deutschland: Status quo - 147

10.2.1 Marktvolumen - 147

10.2.2 Nachfrage nach professioneller Pflege - 148

10.2.3 Angebote der professionellen Pflege - 148

10.3 Ausblick: Der Pflegemarkt als Wachstumsbranche - 150

10.4 Herausforderungen und Chancen in der Langzeitpflege - 153

$10.5 \quad$ Fazit -155

Literatur - 155 


\section{- Zusammenfassung}

Die Alterung der Gesellschaft lässt die Anzahl pflegebedürftiger Menschen weiter steigen. Gleichzeitig wird das Potenzial der Angehörigenpflege zurückgehen, sodass verstärkt auf professionelle Pflegeangebote zurückgegriffen werden muss. Der Pflegemarkt ist und bleibt somit ein Wachstumsmarkt. Die Herausforderung wird es sein, die nötigen personellen und finanziellen Ressourcen zu gewinnen, um das Angebot entsprechend auszuweiten. Die Unternehmen tun daher gut daran, sich für Arbeitskräfte und Kapitalgeber attraktiv aufzustellen. Damit Pflege bezahlbar bleibt, sollte die Politik Planungssicherheit gewährleisten und unternehmerisches Handeln fördern. Parallel können Maßnahmen, welche die Pflegebedürftigkeit verzögern und vermeiden, den Anstieg der Nachfrage nach Pflege abschwächen und die Lebensqualität älterer Menschen steigern.

As a result of the ageing of our society, the number of people in need of care continues to rise. At the same time, the potential of family care will decline, so that professional care options will become more prevalent. Hence, the care market will remain a growth market. The challenge for care providers lies in finding the necessary human and financial resources to expand their range of care services. Companies will need to position themselves as attractive to workers and investors. To ensure that care remains affordable, policy should ensure planning security and encourage entrepreneurial action. At the same time, measures that delay and prevent the need for care can reduce the increase in the demand for care and improve the quality of life of the elderly.

\subsection{Einleitung}

Die Zahl älterer und hochaltriger Menschen in Deutschland nimmt dank steigender Lebenserwartung stetig zu. Mit zunehmendem Alter steigt aber auch die Wahrscheinlichkeit, aufgrund körperlicher oder geistiger Einschränkungen Unterstützung zu benötigen und auf Pflege angewiesen zu sein. Auch wenn Angehörige durch sogenannte informelle Pflegeleistungen aktuell noch den größten Beitrag zur Pflege älterer Menschen leisten, gewinnt die professionelle Pflege immer mehr an Bedeutung. Professionelle Pflege erfolgt dabei oft in Ergänzung zu informeller Pflege, z. B. durch ambulante Dienste, oder aber an Stelle von informeller Pflege, z. B. in Pflegeheimen. Doch in Folge des demographischen Wandels steigt die Anzahl pflegebedürftiger Menschen schneller als die Anzahl verfügbarer pflegender Angehöriger. Anbieter professioneller Pflegeleistungen sehen sich daher einer wachsenden Nachfrage gegenüber. Um der steigenden Nachfrage ein entsprechend steigendes Angebot entgegenzusetzen, bedarf es in erheblichem Umfang zusätzlicher Pflegekräfte sowie zusätzlicher Investitionen in neue Heimplätze.

Dieser Beitrag beleuchtet zunächst die aktuelle Situation des deutschen Pflegemarktes hinsichtlich Marktvolumen, Nachfrage und Angebot ( Abschn. 10.2). Anhand einer Hochrechnung wird anschließend der Pflegebedarf bis zum Jahr 2040 aufgezeigt und mit dem derzeitigen Angebot verglichen ( Abschn. 10.3). Durch diese Gegenüberstellung werden die zukünftigen Kapital- und Personalbedarfe ersichtlich. Da es sich jedoch sowohl bei Personal als auch Kapital um knappe Ressourcen handelt, widmet sich - Abschn. 10.4 detailliert diesen Herausforderungen und diskutiert Lösungsvorschläge, bevor in einem Fazit die wichtigsten Ergebnisse zusammengefasst werden ( $>$ Abschn. 10.5). 


\subsection{Langzeitpflege in Deutschland: Status quo}

\subsubsection{Marktvolumen}

Der Pflegemarkt ist ein Wachstumsmarkt. Im Jahr 2018 betrug das Volumen des deutschen Gesundheitsmarktes rund $391 \mathrm{Mrd}$. $€$ (- Abb. 10.1). Damit stieg der Anteil der Gesundheitsausgaben am Bruttoinlandsprodukt von $11,5 \%$ im Vorjahr auf $11,6 \%$. Er befindet sich damit deutlich über dem Anteil vor der Finanzkrise 2008 (10,5\%). Auf den professionellen Pflegemarkt - die stationäre Pflege in Pflegeheimen sowie die ambulante Pflege durch Pflegedienste - entfielen rund $57 \mathrm{Mrd}$. $€$. Im Vergleich zum Vorjahr ein Zuwachs von $4,4 \%$ oder $2,4 \mathrm{Mrd}$. $€$. Verglichen mit dem Bruttoinlandsprodukt, aber auch im Vergleich mit anderen Gesundheitsausgaben wuchs der Pflegemarkt damit überproportional stark.
Finanziert wurden die Ausgaben für die professionelle Pflege zu zirka der Hälfte durch die soziale Pflegeversicherung (SPV) (- Abb. 10.2). In der stationären Pflege liegt der Anteil bei $46 \%$, in der ambulanten Pflege bei $53 \%$. Dabei wurden für diese Betrachtung die Beiträge der GKV und GUV nicht berücksichtigt. Der Anteil der SPV ist im Vergleich zum Vorjahr in der stationären Pflege leicht zurückgegangen, in der ambulanten Pflege dagegen leicht gestiegen. Private Haushalte finanzieren $41 \%$ der stationären und $37 \%$ der ambulanten Pflege. Damit ist der privat finanzierte Anteil in der stationären Pflege seit 2006 nahezu unverändert, wohingegen er in der ambulanten Pflege tendenziell leicht rückläufig ist. Steigende Lohn- und Investitionskosten werden jedoch zukünftig den Eigenanteil steigen lassen, sofern die Pflegesätze nicht in entsprechendem Maße angepasst werden.

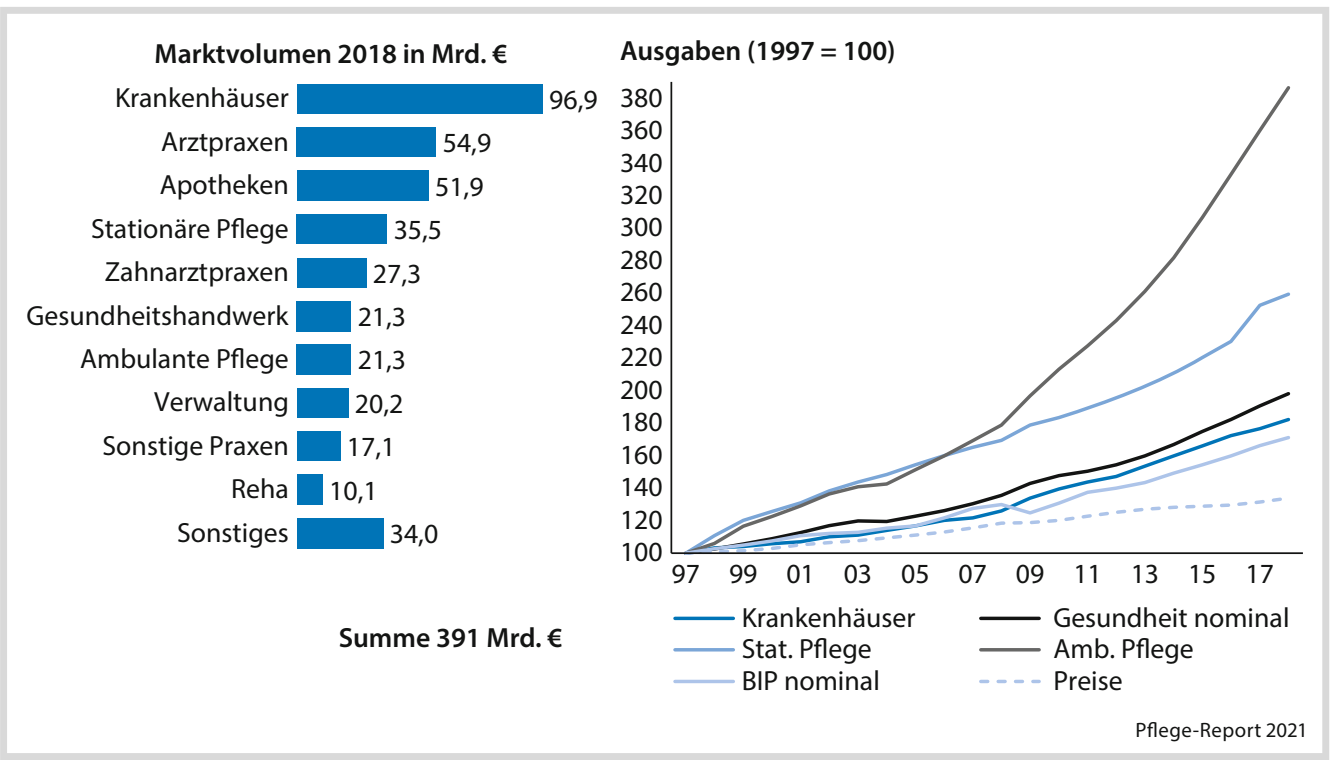

- Abb. 10.1 Marktvolumen 2018 und Änderung über die Zeit (Quelle: RWI; Statistisches Bundesamt 2020a, 2020c, 2020d) 


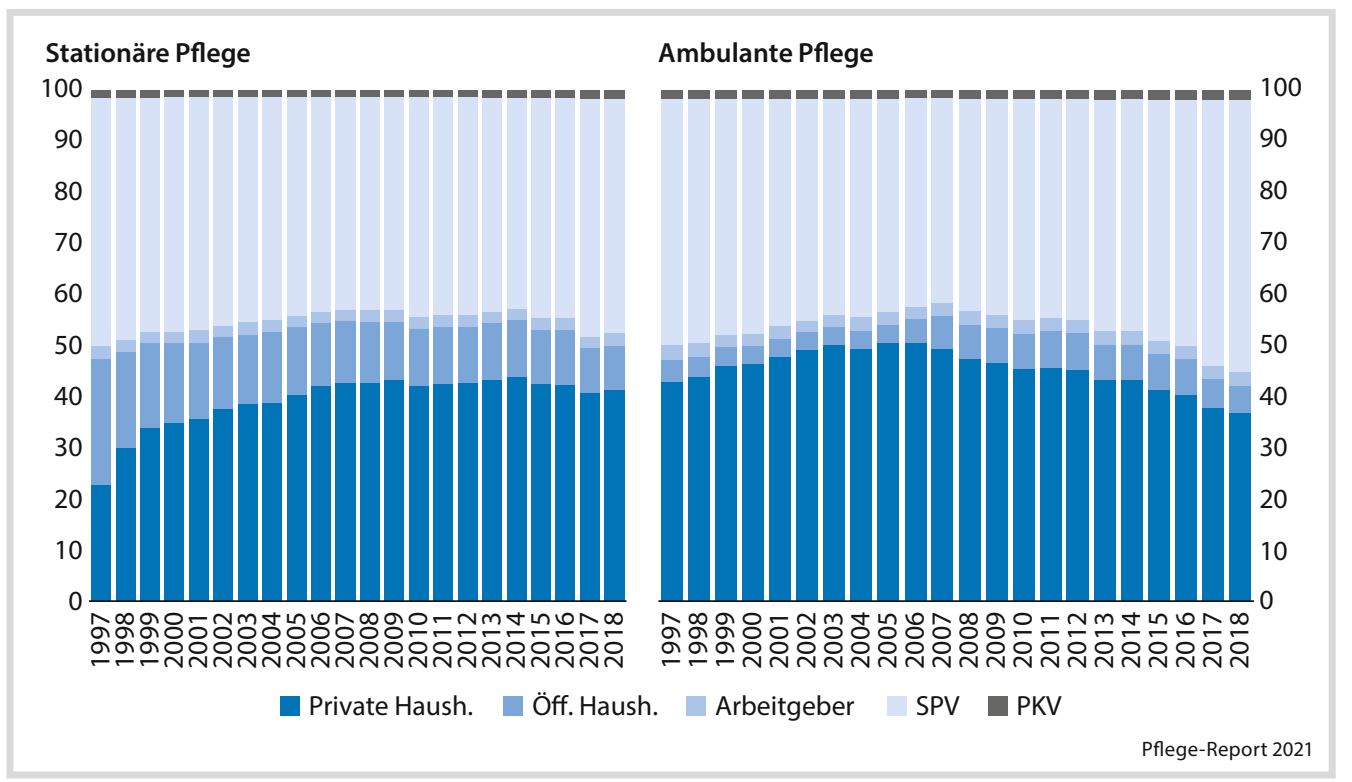

- Abb. 10.2 Kostenträger der stationären und ambulanten Pflege (Anteile in \%) (Quelle: RWI; Statistisches Bundesamt 2020b)

\subsubsection{Nachfrage nach professioneller Pflege}

Im Jahr 2017 gab es laut Pflegestatistik 3,4 Mio. pflegebedürftige Menschen. ${ }^{1}$ Davon wurden jeweils knapp ein Viertel $(24 \%)$ vollstationär und durch ambulante Dienste versorgt, etwas mehr als die Hälfte erhielt Pflegegeld. - Abb. 10.3 zeigt die Verteilung der Pflegebedürftigen nach Art der Pflege. Insbesondere die Ausweitung des Pflegebedürftigkeitsbegriffs führte $2017 \mathrm{zu}$ einem starken Anstieg der Pflegegeldempfänger. Damit liegt ihr Anteil leicht höher als 1999. Der Anteil ambulant betreuter Menschen nahm über die Zeit

1 In der Pflegestatistik konnten geschätzt 100.000 Pflegebedürftige des Pflegegrades 1 nicht erfasst werden, die keine Leistungen der ambulanten Pflegedienste oder Pflegeheime bzw. ausschließlich Leistungen der nach Landesrecht anerkannten Angebote zur Unterstützung im Alltag erhalten (Statistisches Bundesamt 2019). Die Prognosen in $>$ Abschn. 10.3 sind um diese Zahl korrigiert. ebenfalls zu, während der Anteil - nicht aber die Anzahl - stationär Versorgter zurückging.

\subsubsection{Angebote der professionellen Pflege}

Mit wachsender Nachfrage nach Pflegeleistungen stieg auch das Angebot. Das professionelle Pflegeangebot umfasste im Jahr 2017 insgesamt 952.000 Plätze in 14.480 stationären Heimen und 14.050 ambulante Dienste (- Abb. 10.4). Betrachtet man neu entstandene stationäre Plätze, hat sich das Angebotswachstum seit 2009 jedoch stetig verlangsamt.

Das Angebot ist zwischen privaten, freigemeinnützigen und öffentlich-rechtlichen Anbietern aufgeteilt. Gemessen an der Anzahl betreuter Menschen machen öffentlich-rechtliche Anbieter nur $6 \%$ des stationären und $2 \%$ des ambulanten Angebots aus (• Abb. 10.5). Im stationären Bereich werden die meisten Pflegebedürftigen $(55 \%)$ in freigemeinnützigen Heimen betreut, $39 \%$ in privaten Heimen. 


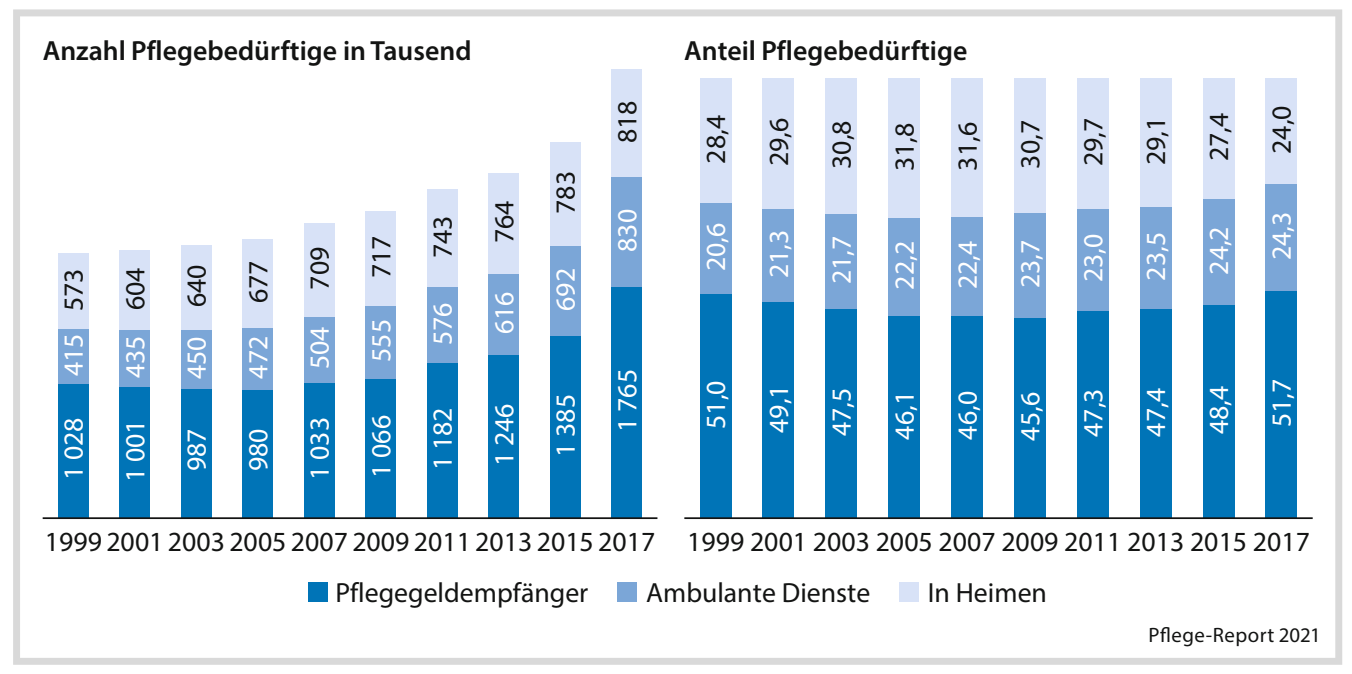

- Abb. 10.3 Verteilung der Zahl der Pflegebedürftigen nach Art der Pflege in \% (Quelle: RWI; Heger et al. 2019) Anmerkung: Angaben zu Pflegebedürftigen in Heimen beziehen sich analog zur Pflegestatistik ab 2009 nur noch auf vollstationär Pflegebedürftige

$$
\begin{aligned}
& \text { Anzahl Einrichtungen }{ }^{1} \text { in Tsd. } \\
& \begin{array}{llllllllll}
10,8 & 10,6 & 10,6 & 11,0 & 11,5 & 12,0 & 12,3 & 12,7 & 13,3 & 14,1 \\
\hline 8,9 & 9,2 & 9,7 & 10,4 & 11,0 & 11,6 & 12,4 & 13,0 & 13,6 & 14,5
\end{array}
\end{aligned}
$$$$
\overline{1999200120032005200720092011201320152017}
$$$$
\text { - Ambulant Stationär }
$$

Markteintritte stationäre Einrichtungen p.a. ${ }^{2}$

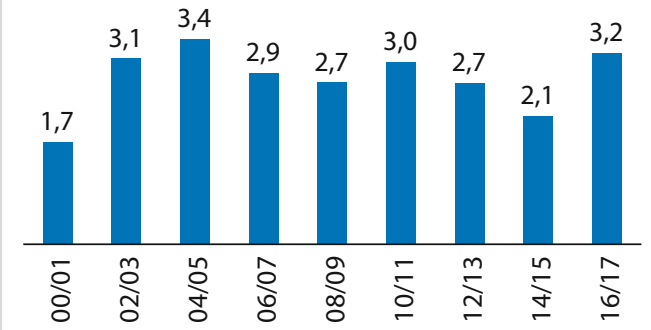

\section{Anzahl Plätze ${ }^{1}$ in Tsd.}

$\longdiv { 6 4 5 \quad 6 7 4 \quad 7 1 3 \quad 7 5 7 \quad 7 9 9 \quad 8 4 5 \quad 8 7 6 \quad 9 0 3 \quad 9 2 9 9 5 2 }$

1999200120032005200720092011201320152017

- Stationär

Markteintritte stationäre Plätze p.a. ${ }^{2}$

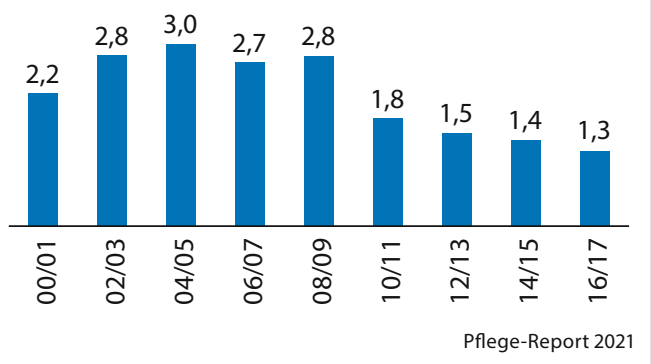

- Abb. 10.4 Änderung der Zahl der Einrichtungen und Plätze in \% (Quelle: Heger et al. 2019) Anmerkung: ${ }^{1}$ Alle Heime ${ }^{2}$ Interpolation auf die einzelnen Jahre

Dagegen machen bei den ambulanten Diensten private Anbieter mit 52\% mittlerweile die Mehrheit aus. 


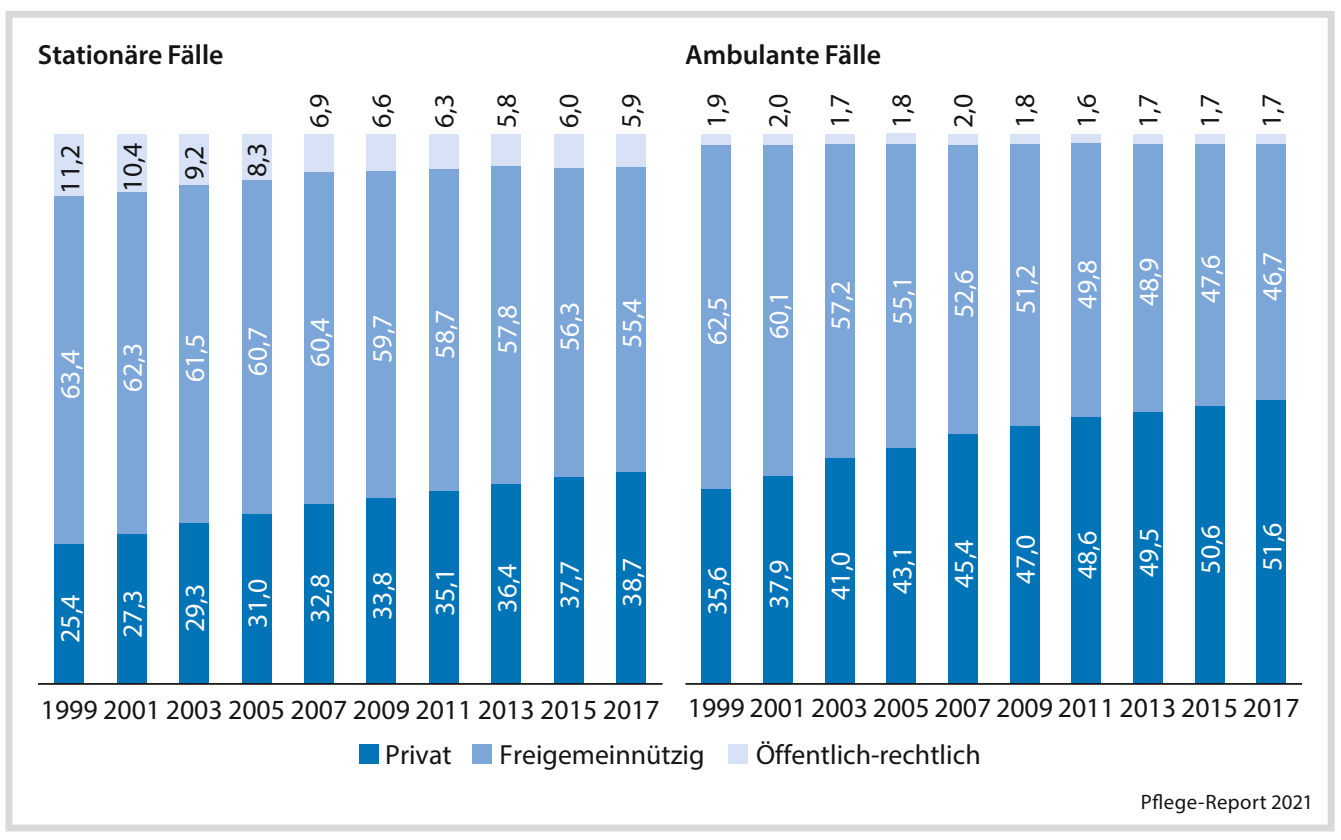

- Abb. 10.5 Verteilung der Pflegebedürftigen nach Trägerschaft (Anteil in \%) (Quelle: Heger et al. 2019)

Anmerkung: Zahlen bis 2015 inkl. Pflegebedürftige der Stufe 0. Pflegebedürftige mit unbekannter Pflegestufe wurden bis 2015 Stufe II und ab 2017 Pflegegrad 3 zugeordnet

\subsection{Ausblick: Der Pflegemarkt als Wachstumsbranche}

Die zunehmende Nachfrage nach Pflegeleistungen ist zum überwiegenden Teil durch die demographische Entwicklung bestimmt, da die Pflegewahrscheinlichkeit mit steigendem Alter stark zunimmt. So ist die Wahrscheinlichkeit auf Pflege angewiesen zu sein für unter 70-jährige noch relativ gering (• Abb. 10.6). Zwischen 80 und 85 Jahren liegt die Pflegewahrscheinlichkeit schon bei $30 \%$ für Frauen und $23 \%$ für Männer. Über 90-jährige Frauen haben bereits eine 78-prozentige Wahrscheinlichkeit Pflege zu benötigen. Bei Männern liegt sie immerhin bei $68 \%$. Da gerade die Altersgruppen mit hohem Pflegebedarf in den kommenden Jahrzehnten stark zunehmen werden, dürfte sich diese Entwicklung auch in der Nachfrage nach Pflegeleistungen widerspiegeln. Der beobachtete Anstieg der Pflegequo- ten innerhalb einer Altersgruppe über die Zeit bedeutet jedoch nicht zwangsläufig eine Verschlechterung des allgemeinen Gesundheitszustandes. Vielmehr wurde der Kreis der Leistungsempfänger ausgeweitet, um insbesondere auch Menschen mit kognitiven Einschränkungen $\mathrm{zu}$ erfassen. Am deutlichsten war dies mit der Einführung des neuen Pflegebedürftigkeitsbegriffs der Fall, weshalb ein Anstieg der Pflegequoten insbesondere bei den Hochaltrigen zu verzeichnen ist.

Eine Hochrechnung auf Basis der aktuellen Pflegequoten des Jahres 2017 und Bevölkerungsprognosen für Deutschland bis zum Jahr 2040 ergibt einen Anstieg der Zahl der Pflegebedürftigen um $42 \%$ auf 4,98 Mio. (- Abb. 10.7). ${ }^{2}$ Die Zahl der in Heimen vollstationär versorgten Pflegebedürftigen wächst am stärksten um $52 \%$ auf 1,25 Mio., die Zahl

2 Die Prognosen basieren auf dem Pflegeheim Rating Report 2020 (Heger et al. 2019). 


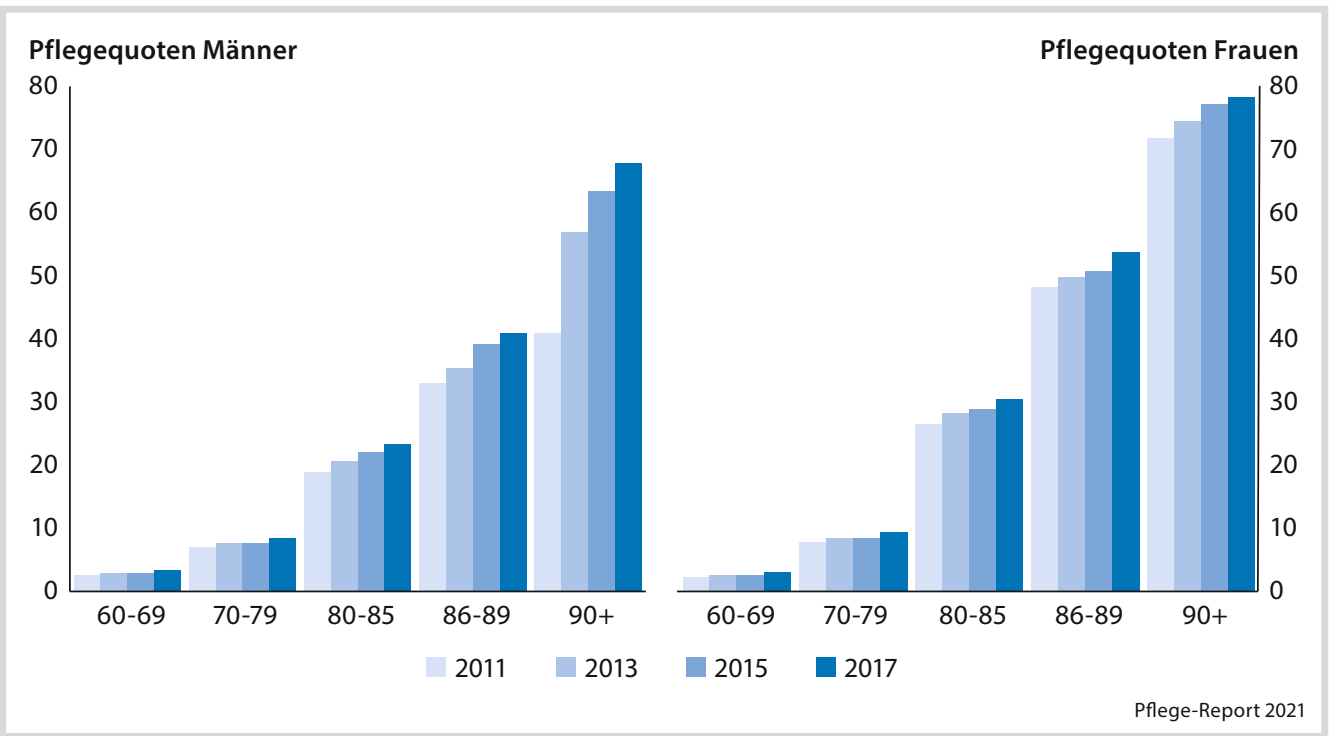

- Abb. 10.6 Pflegequoten insgesamt - stationär, ambulant und Pflegegeldempfänger (in \%) (Quelle: Heger et al. 2019) Anmerkung: 2013-2015 inkl. Pflegebedürftige der Stufe 0; ab 2017 Pflegebedürftigkeit nach neuem Pflegebedürftigkeitsbegriff

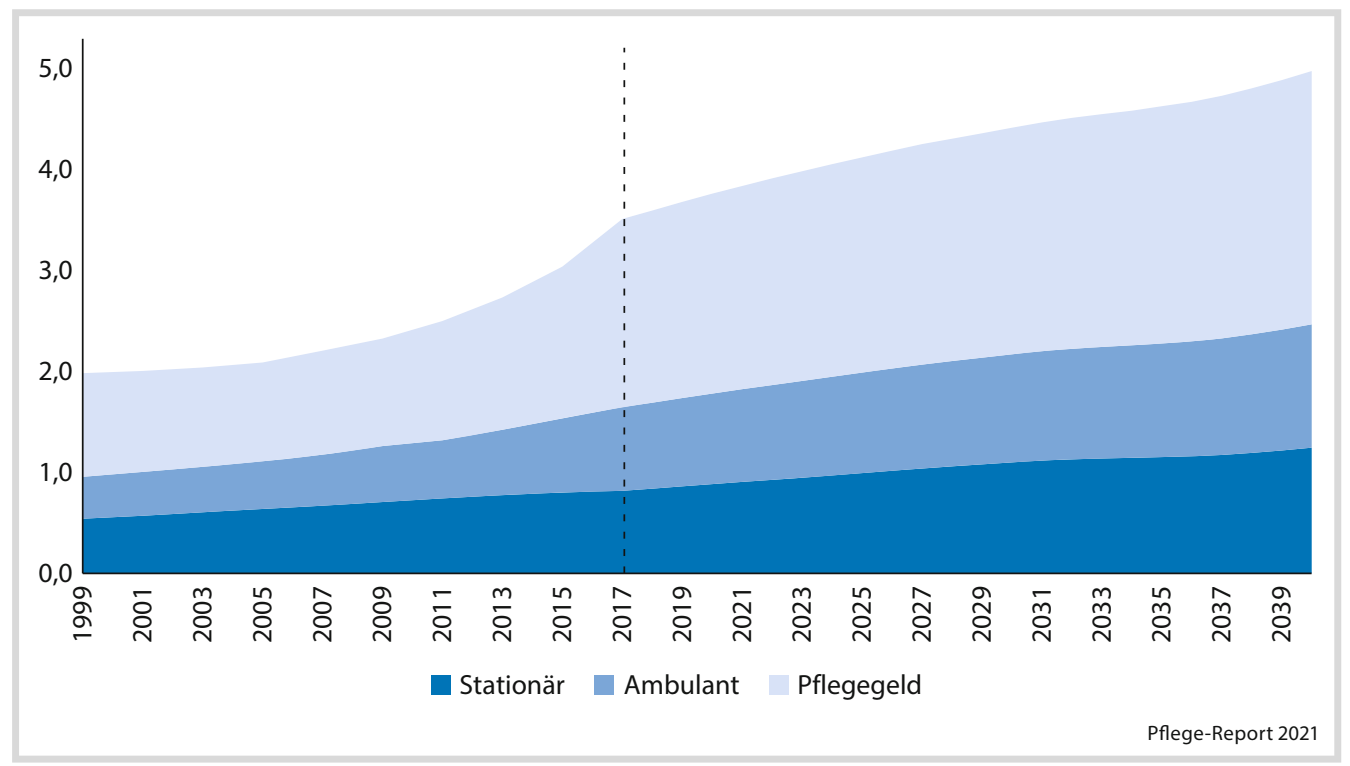

- Abb. 10.7 Zahl der Pflegebedürftigen nach Pflegeart (in Mio.) (Quelle: RWI; FDZ der Länder 2009; 2011; 2017; 2019; FDZ Ruhr am RWI 2017)

Anmerkung: 2013-2015 inkl. Pflegebedürftige der Stufe 0; ab 2017 Pflegebedürftigkeit nach neuem Pflegebedürftigkeitsbegriff. Zum Ausgleich nicht erfasster Leistungsempfänger nach Landesrecht werden 2017 100.000 Pflegegeldempfänger ergänzt 


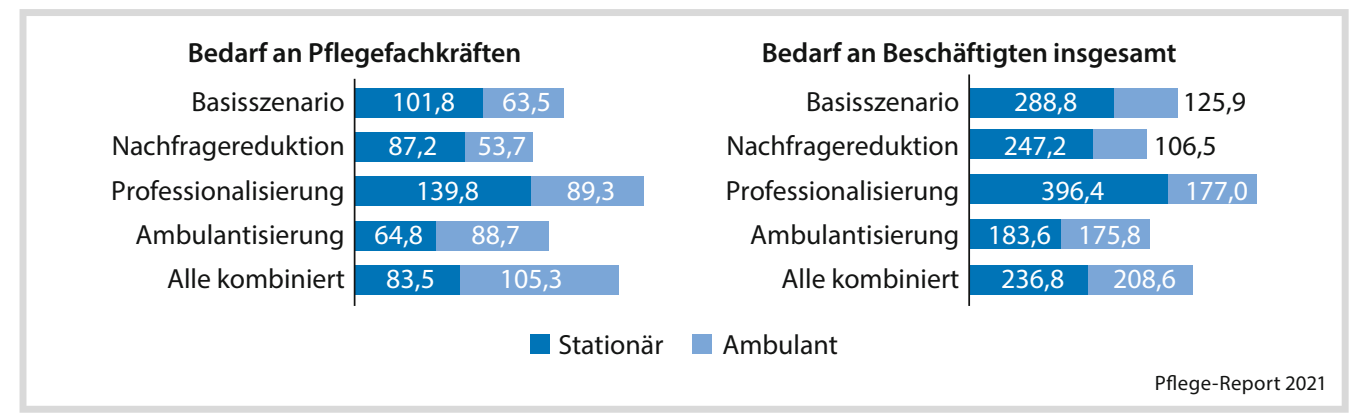

- Abb. 10.8 Bedarf an zusätzlichen Pflegefachkräften und Beschäftigten insgesamt (2017 bis 2040; in Tsd. Vollkräften). (Quelle: Heger et al. 2019)

Anmerkung: Pflegefachkräfte umfasst staatlich anerkannte/-r Altenpfleger/-in, Gesundheits- und Krankenpfleger/-in, Gesundheits- und Kinderkrankenpfleger/-in, Heilerziehungspfleger/-in und Heilerzieher/-in, Abschluss einer pflegewissenschaftlichen Ausbildung an einer Fachhochschule oder Universität

ambulant gepflegter Menschen um $47 \%$ auf 1,22 Mio. Etwas geringer fällt der Anstieg der Zahl der Pflegegeldempfänger aus. Diese steigt um $35 \%$ auf 2,51 Mio.

Die Prognosen spiegeln dabei allein den Effekt der Bevölkerungsentwicklung wider. Es ist jedoch denkbar, dass sich die Pflegewahrscheinlichkeit oder das Nachfrageverhalten der pflegebedürftigen Menschen über die Zeit verändert. So kann sich der allgemeine Gesundheitszustand der Bevölkerung verbessern, wodurch sich der Pflegeeintritt verzögert oder die Pflegedauer reduziert. Die Folge wäre eine Reduktion der Nachfrage nach Pflegeleistungen. Denkbar ist auch eine Verschiebung der Nachfrage hin zu mehr professioneller Pflege, weil zukünftig weniger Angehörige als potenzielle Pflegekräfte zur Verfügung stehen, sowie eine Verschiebung hin zu mehr ambulanter Pflege, da ein möglichst langer Verbleib in den eigenen vier Wänden oft dem Wunsch der pflegebedürftigen Menschen entspricht (PwC 2017). Eine vollständige Beschreibung dieser verschiedenen Szenarien (Basisszenario, Nachfragereduktion, Professionalisierung und Ambulantisierung) findet sich in Heger et al. (2019). Während eine zunehmende Professionalisierung oder Ambulantisierung zwar die Verteilung der Pflegebedürftigen nach Versorgungsart beeinflussen würde, hat nur eine Nachfragereduktion auch Einfluss auf die Ge- samtzahl der Pflegebedürftigen. Würde sich die Nachfrage z. B. um $5 \%$ reduzieren, läge die Zahl der Pflegebedürftigen 2040 nur mehr bei 4,73 Mio.

Je nachdem, ob es zu einer Nachfragereduktion, einer Professionalisierung oder einer Ambulantisierung kommt, oder ob alle Veränderungen gemeinsam auftreten, fällt der Zuwachs an Pflegebedürftigen in den einzelnen Pflegearten mehr oder weniger stark aus. So reichen die Prognosen für die Zahl der stationär Pflegebedürftigen von 1,09 Mio. bei zunehmender Ambulantisierung bis hin zu 1,41 Mio. bei steigender Professionalisierung. Der projizierte Zuwachs im ambulanten Bereich reicht von 1,16 Mio. bei einer Nachfragereduktion bis zu 1,48 Mio. im kombinierten Szenario. Die Anzahl der Pflegegeldempfänger variiert von 2,51 Mio. im kombinierten Szenario bis 2,08 Mio. in einem Basisszenario, wenn nur die Bevölkerungsentwicklung berücksichtigt wird. Entsprechend wird auch mehr oder weniger zusätzliches Personal und Kapital benötigt. Aber auch im Falle einer Nachfragesenkung wird die Nachfrage nach Pflegeleistungen absolut gesehen weiterhin wachsen, wenn auch bei geringerer Wachstumsrate.

Ausgehend von 2017 zeigt - Abb. 10.8 den zu erwartenden zusätzlichen Personalbedarf bis 2040 bei gleichbleibendem Betreu- 


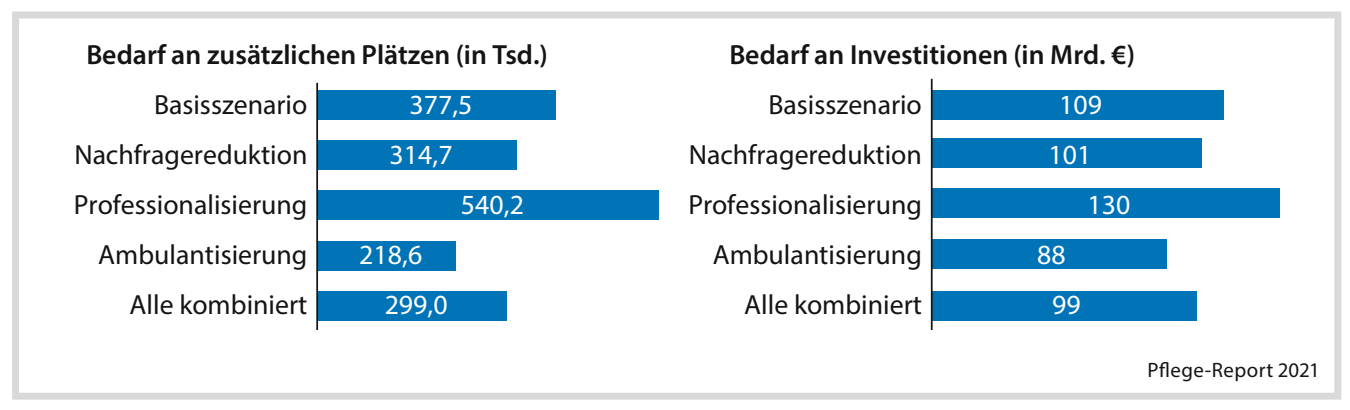

- Abb. 10.9 Bedarf an Plätzen und Investitionen (2017 bis 2040) (Quelle: RWI; Heger et al. 2019) Anmerkung: Investitionen umfassen Neu- und Reinvestitionen

ungsverhältnis. Demzufolge sind im Basisszenario gut 165.000 zusätzliche Pflegefachkräfte erforderlich. Insgesamt ergibt sich sogar ein Personalbedarf von knapp 415.000 Vollkräften.

Zusätzlich zum Personalbedarf werden weitere stationäre Plätze benötigt. Auch im Fall einer Nachfragereduktion ergibt sich ein Bedarf an zusätzlichen vollstationären Plätzen von knapp 315 Tsd. Plätzen (• Abb. 10.9). Ohne Nachfragereduktion beläuft sich der Platzbedarf sogar auf 378.000 neue Plätze. Zur Finanzierung dieser Plätze sowie zur notwendigen Sanierung existierender Plätze, ergibt sich zwischen 2017 und 2040 dadurch ein Kapitalbedarf zwischen $88 \mathrm{Mrd}$. $€$ im Falle einer zunehmenden Ambulantisierung und $130 \mathrm{Mrd}$. $€$ im Falle einer zunehmenden Professionalisierung.

\subsection{Herausforderungen und Chancen in der Langzeitpflege}

Die Prognose des Pflegebedarfs hat die Notwendigkeit von zusätzlichem Pflegeangebot deutlich gezeigt. Gegen Ende der 20er Jahre schwächt sich der demographiebedingte Wachstumstrend zwar leicht ab, nimmt Ende der 30er Jahre jedoch wieder zu. Die steigende Nachfrage, die aus Anbietersicht zunächst positiv zu bewerten ist, birgt aber auch
Herausforderungen. So fällt es professionellen Pflegeanbietern immer schwerer, ausreichend qualifizierte Pflegekräfte am Markt zu finden. Zwar steigen die Löhne für Pflegekräfte aufgrund des Fachkräftemangels bereits schneller als in anderen Branchen. Doch die hohe Arbeitsbelastung, familienunfreundliche (Schicht-)Arbeitszeiten und geringe Aufstiegschancen reduzieren die Attraktivität des Pflegeberufs. Und auch der besser zahlende Krankenhaussektor ist auf der Suche nach Pflegekräften. Die generalistische Pflegeausbildung dürfte dieses Problem noch weiter verschärfen, statt es wie erhofft abzuschwächen. Hinzu kommt, dass sich die Pflegebranche - wie alle anderen Branchen auch - einer schrumpfenden Arbeitsbevölkerung gegenübersieht. Die Pflegebranche konkurriert daher nicht nur mit dem Krankenhaussektor um neue Auszubildende, sondern mit allen Branchen. Eine Steigerung der Attraktivität des Pflegeberufs sowie die Anwerbung von Fachkräften aus dem Ausland helfen dabei, mehr Personal zu gewinnen. Diese Maßnahmen werden aber nicht ausreichen. Vielmehr muss es gelingen, den notwendigen Personaleinsatz zu reduzieren, ohne die Qualität der Pflege zu verringern. Ein differenzierterer und heimspezifischer Personalmix von Pflegefach- und Pflegehilfskräften entsprechend den anfallenden Tätigkeiten könnte zumindest den Bedarf an Pflegefachkräften dort, wo der größte Mangel herrscht, etwas lindern. Dazu muss sich der Qualifikationsmix jedoch an den Bedürfnissen der 
versorgten Personen ausrichten und nicht an starren Fachkraftquoten. So zeigt z. B. die Soll-Wert-Berechnung für einen angemessenen Personalbedarf insbesondere einen Mehrbedarf an Assistenzkräften (Rothgang 2020). Aber auch technische und IT-basierte Lösungen können dazu beitragen, die Pflegekräfte zu entlasten. Damit aber ein Anreiz für innovative Lösungsansätze existiert, müssen Personalvorgaben flexibel genug sein, um solche Lösungen zu erlauben. Gleichzeitig muss die Refinanzierbarkeit sowohl der Anfangsinvestitionen als auch der laufenden Kosten gesichert sein, wobei auf Kosteneffizienz der Maßnahmen $\mathrm{zu}$ achten ist. Lediglich eine Bezahlung nach Tarif einzuführen, ohne die anderen Probleme anzugehen, führt zu einer Verteuerung der Pflege und wird das Fachkräfteproblem nicht lösen.

Die hohe Regulierungsdichte schränkt dabei nicht nur im Personalbereich den unternehmerischen Handlungsspielraum der Pflegeanbieter stark ein. Durch die nach Bundesland oft unterschiedlichen Regelungen zu Personal sowie bauliche Vorgaben wird auch die Gewinnung von privatem Kapital erschwert. Denn die mit der Regulierung verbundenen Kosten sowie fehlende Planungssicherheit bei sich ändernden Vorgaben schreckt Investoren ab. Privates Kapital fließt dann in andere Bereiche und Investitionen werden verhindert. Hinzu kommt, dass sich die meisten Bundesländer bisher nur geringfügig an der Finanzierung von Investitionskosten in Pflegeheimen beteiligen (KPMG 2020). Privates Kapital ist deshalb neben öffentlichem und freigemeinnützigem Kapital dringend nötig, um den hohen Investitionsbedarf zu bewältigen. Hohe Einbettzimmerquoten vergrößern den Investitionsbedarf weiter. Sind die notwendigen Umbauten nicht rentabel, könnten sogar noch weitere Plätze wegfallen. Steht der wachsenden Nachfrage nach Pflege aber kein ausreichendes Angebot gegenüber, können pflegebedürftige Menschen nicht das für sie passendste Angebot auswählen. Ein Wettbewerb um gute Pflege bei günstigen Preisen findet nicht statt und es bestünde ein Anreiz, nur ein Minimum an Pflegequa- lität anzubieten. Eine Reduktion der Regulierung auf Vorgaben zu (Ergebnis-)Qualität wäre daher einer zu detaillierten Vorgabenliste vorzuziehen.

Höhere Löhne für Pflege(fach)kräfte sowie der hohe Investitionsbedarf werden die Pflege verteuern. Damit gute Pflege bezahlbar bleibt, darf das Ziel nicht nur sein, neues Personal und Kapital zu gewinnen. Vielmehr muss auch die Reduktion des Personal- und Kapitalbedarfs angestrebt werden. Idealerweise würde man dies durch die Vermeidung und Verzögerung von Pflegebedürftigkeit erreichen. Dazu kann der medizinische Fortschritt beitragen, wenn Altersleiden geheilt oder gelindert werden können und Pflegebedürftigkeit damit erst später im Leben und für kürzere Zeit auftritt - eine sogenannte Kompression der Morbidität. Allerdings führt medizinischer Fortschritt nicht immer zu einer Kompression, sondern in Teilen sogar zu einer Expansion der Morbidität, wenn Krankheiten zwar behandelt, aber nicht geheilt werden können. Daher muss vermehrt in die Prävention - wie Reha vor sowie Reha bei Pflege - investiert werden. Dies wird immer wieder gefordert. Das zugrunde liegende Anreizproblem, dass eine Vermeidung von SPV-Leistungen auf Kosten von GKV-Leistungen aus Kassensicht nicht erstrebenswert ist, wurde jedoch noch nicht gelöst. Auch eine engere Abstimmung zwischen einzelnen Gesundheitsdienstleistern, wie es in Modellprojekten vermehrt erprobt wird (z. B. „Gesundes Kinzigtal"), kann die gesundheitliche Versorgung passgenauer machen, Wartezeiten auf die richtige medizinische, pflegerische oder rehabilitative Maßnahme verkürzen und daher die Gesundheit und Lebensqualität der Menschen verbessern. Auch neue Versorgungsformen sollten dabei berücksichtigt werden, denn die Trennung zwischen ambulant und stationär verschwimmt insbesondere im Pflegebereich immer mehr.

Zum Erhalt eines möglichst selbstbestimmten Lebens sollte Prävention allerdings nicht erst bei drohender Pflegebedürftigkeit greifen. Es gilt auch zu prüfen, ob Anreize für einen gesunden Lebensstil sowie frühzei- 
tige Rehabilitationsmaßnahmen in ausreichendem Maße durchgeführt werden. Zeichnen sich Einschränkungen ab, können technische Hilfsmittel die Mobilität unterstützen oder ITLösungen helfen den Alltag einer immer Technik- und IT-affineren älteren Bevölkerung zu organisieren.

\subsection{Fazit}

Durch die Alterung der Gesellschaft und den zunehmenden Trend hin zu mehr professioneller Pflege wird der Pflegemarkt weiterhin stark wachsen. Durch die steigende Nachfrage ergibt sich ein deutlicher Mehrbedarf an Personal und Kapitel, wie die Prognosen aus Abschn. 10.3 gezeigt haben. Anbieter professioneller Pflegeleistungen können die Chancen des Wachstumsmarkts Pflege daher nur nutzen und der wachsenden Nachfrage mit einem entsprechend wachsenden Angebot begegnen, wenn sie ausreichend Pflegekräfte und Kapital gewinnen können. Ohne verbesserte Rahmenbedingungen wird dies nicht gelingen.

Um den notwendigen Kapitalbedarf zu decken, wird man sowohl privates als auch nichtprivates Kapital benötigen. Viele, sich häufig ändernde oder regional verschiedene Regulierungen schrecken jedoch insbesondere private Investoren leicht ab und können das Angebot reduzieren. Ein unzureichendes Angebot geht aber zu Lasten der Pflegebedürftigen. Von einem Wettbewerb um gute Qualität und Preise würden pflegebedürftige Menschen dagegen profitieren. So ist eine gezielte Festlegung der (Ergebnis-)Qualität der Pflege Einschränkungen der unternehmerischen Handlungsfreiheit vorzuziehen.

Eine bessere Bezahlung von Pflegekräften wird in der aktuellen Situation mit einer kleiner werdenden Arbeitsbevölkerung nicht ausreichen. Auch weiche Faktoren wie Karrieremöglichkeiten, Vereinbarkeit von Familie und Beruf sowie die hohe physische und psychische Belastung müssen sich verbessern. Der Personaleinsatz sollte zudem flexibler ge- staltbar sein und technische Lösungen, die Arbeitsabläufe optimieren und den Personalbedarf reduzieren können, müssen bei der Pflegefinanzierung Berücksichtigung finden.

Nicht zuletzt sollten künftige Konzepte verstärkt die Reduktion der Nachfrage in den Fokus nehmen. Präventive Maßnahmen wie Reha vor und bei Pflege, die die Pflegebedürftigkeit verhindern oder zumindest verzögern können, sollten deutlich gesteigert werden. Gleiches gilt für die Förderung eines gesunden Lebensstils von Anfang an.

\section{Literatur}

FDZ der Länder (2009) Forschungsdatenzentren der Statistischen Ämter des Bundes und der Länder, Nutzung von Mikrodaten der Pflegestatistiken 1999-2009, Projektnummer 1559-2010

FDZ der Länder (2011) Forschungsdatenzentren der Statistischen Ämter des Bundes und der Länder, Nutzung von Mikrodaten der Pflegestatistiken 2009-2011, Projektnummer 2237-2013

FDZ der Länder (2017) Nutzung von Mikrodaten der Pflegestatistiken 2013-2015, Projektnummer 3067-2015

FDZ der Länder (2019) Forschungsdatenzentren der Statistischen Ämter des Bundes und der Länder, Nutzung von Mikrodaten der Pflegestatistiken 2015-2017, Projektnummer 3880-2019

FDZ Ruhr am RWI (2017) Population Forecast. RWIGEO-GRID. Version: 1. RWI - Leibniz-Institut für Wirtschaftsforschung. (Datensatz)

Heger D, Augurzky B, Kolodziej I, Krolop S, Wuckel C (2019) Pflegeheim Rating Report 2020: Zwischen Nachfragewachstum und Kostendruck. medhochzwei, Heidelberg

KPMG (KPMG Law Rechtsanwaltsgesellschaft mbH und die KPMG AG Wirtschaftsprüfungsgesellschaft) (2020) Studie zur Umsetzung der Berichtspflicht der Länder zu Investitionskosten in Pflegeeinrichtungen

PwC (PricewaterhouseCoopers GmbH WPG) (2017) Berichtsband „Bevölkerungsbefragung Pflege in Deutschland Dezember 2017“"

Rothgang H (2020) Abschlussbericht im Projekt Entwicklung eines wissenschaftlich fundierten Verfahrens zur einheitlichen Bemessung des Personalbedarfs in Pflegeeinrichtungen nach qualitativen und quantitativen Maßstäben gemäß § 113c SGB XI (PeBeM)

Statistisches Bundesamt (Hrsg) (2019) Pflegestatistik Pflege im Rahmen der Pflegeversicherung - Deutschlandergebnisse - 2017. Statistisches Bundesamt, Wiesbaden 
Statistisches Bundesamt (Hrsg) (2020a) Gesundheitsausgaben: Deutschland, Jahre, Einrichtungen. Statistisches Bundesamt, Wiesbaden

Statistisches Bundesamt (Hrsg) (2020b) Gesundheitsausgaben: Deutschland, Jahre, Ausgabenträger, Leistungsarten, Einrichtungen. Statistisches Bundesamt, Wiesbaden
Statistisches Bundesamt (Hrsg) (2020c) Verbraucherpreisindex (inkl. Veränderungsraten): Deutschland, Jahre. Statistisches Bundesamt, Wiesbaden

Statistisches Bundesamt (Hrsg) (2020d) VGR des Bundes - Bruttowertschöpfung, Bruttoinlandsprodukt. Statistisches Bundesamt, Wiesbaden

Open Access Dieses Kapitel wird unter der Creative Commons Namensnennung 4.0 International Lizenz (http:// creativecommons.org/licenses/by/4.0/deed.de) veröffentlicht, welche die Nutzung, Vervielfältigung, Bearbeitung, Verbreitung und Wiedergabe in jeglichem Medium und Format erlaubt, sofern Sie den/die ursprünglichen Autor(en) und die Quelle ordnungsgemäß nennen, einen Link zur Creative Commons Lizenz beifügen und angeben, ob Änderungen vorgenommen wurden.

Die in diesem Kapitel enthaltenen Bilder und sonstiges Drittmaterial unterliegen ebenfalls der genannten Creative Commons Lizenz, sofern sich aus der Abbildungslegende nichts anderes ergibt. Sofern das betreffende Material nicht unter der genannten Creative Commons Lizenz steht und die betreffende Handlung nicht nach gesetzlichen Vorschriften erlaubt ist, ist für die oben aufgeführten Weiterverwendungen des Materials die Einwilligung des jeweiligen Rechteinhabers einzuholen. 